EPJ Web of Conferences 49, 17010 (2013)

DOI: $10.1051 /$ epjconf/20134917010

(C) Owned by the authors, published by EDP Sciences, 2013

\title{
Probing non-standard top-quark couplings via optimal-observable analyses at LHC
}

\author{
Zenrō Hioki ${ }^{1, a}$ and Kazumasa Ohkuma ${ }^{2, b}$ \\ ${ }^{1}$ Institute of Theoretical Physics, University of Tokushima, Tokushima 770-8502, Japan \\ ${ }^{2}$ Department of Information Science, Fukui University of Technology, Fukui 910-8505, Japan
}

\begin{abstract}
Focusing attention on top-quark pair production and its decay processes at the LHC, non-standard top-quark couplings are studied based on the effective Lagrangian constructed with $S U(3) \times S U(2) \times U(1)$ invariant dimension- 6 operators. The optimal-observable analysis is carried out for the charged-lepton distributions in $p p \rightarrow t \bar{t} X \rightarrow \ell^{+} X^{\prime}(\ell=e$ or $\mu$ ) in order to estimate the expected statistical uncertainties in measurements of those non-standard $t \bar{t} g$ and $t b W$ couplings that contribute to this process in the leading order.
\end{abstract}

\section{Introduction}

Almost twenty years have passed since the top quark was discovered at the Tevatron [1,2]. Even today, however, this quark is still the heaviest in the experimentallyconfirmed elementary particles. Therefore, precise measurements of the top quark are expected to be one of the most promising ways for finding possible signals from new physics beyond the standard model in the current situation where there has been no discovery of non-standard particles [3]. The Large Hadron Collider (LHC) is now the unique facility for those top-quark studies in stead of the Tevatron.

In any processes, non-standard signals mean nonnegligible differences between actual experimental data and the corresponding standard-model prediction. Such deviations might be originated from quantum effects of new particles which are not belonging to the standardmodel framework. Those quantum effects are usually characterized as new form factors corresponding to the non-standard couplings. The size of the form factors are calculable if you take a specific model which is an extension of the standard model. We are also able to treat those form factors as parameters in the manner of effectiveLagrangian approach, if we would like to perform analyses as model-independently as possible. The latter is the strategy which we have been taking for our new-physics search in top-couplings [4-6].

In this note, we focus on our latest work [6] and show the studies on the top-quark production and its semileptonic decay process $p p \rightarrow t \bar{t} X \rightarrow \ell^{+} X^{\prime}(\ell=e$ or $\mu)$ at the LHC aiming to estimate the statistical significance of measurable non-standard top-quark couplings. Here, non-standard interactions which are originated from the

\footnotetext{
ae-mail: hioki@ias.tokushima-u.ac.jp

be-mail: ohkuma@fukui-ut.ac.jp
}

quantum effects of non-standard particles are described by $S U(3) \times S U(2) \times U(1)$ invariant dimension- 6 operators, and parameterized as non-standard top couplings. The statistical significances are estimated through the optimalobservable analysis.

\section{Strategy}

In this section, we explain briefly the effective Lagrangian and the optimal-observable analysis, both of which play important roles in our analysis.

\subsection{Effective Lagrangian}

The effective-Lagrangian approach, in which its lowenergy form reproduces the standard model, is one of the most general methods to describe new-physics phenomena when the energy of our experimental facility is not high enough to produce new particles. Assuming any nonstandard-model particles too heavy to appear as real ones, the effective Lagrangian is given as

$$
\mathcal{L}_{\text {eff }}=\mathcal{L}_{\mathrm{SM}}+\frac{1}{\Lambda^{2}} \sum_{i}\left(C_{i} O_{i}+C_{i}^{*} O_{i}^{\dagger}\right),
$$

where $\mathcal{L}_{\mathrm{SM}}$ is the standard-model Lagrangian, $O_{i}$ mean $S U(3) \times S U(2) \times U(1)$ gauge-invariant operators of massdimension 6 involving only the standard-model fields [7, 8] and their coefficients $C_{i}$ parameterize quantum effects of new particles at an energy less than the assumed newphysics scale $\Lambda .{ }^{1}$ In this framework, all the form factors related to $C_{i}$ are dealt with as constant parameters, without supposing any specific new-physics models.

\footnotetext{
${ }^{1}$ Since dimension-5 operators violate the lepton-number conservation, they are not treated hereafter. Therefore, we deal with dimension-6 operators, which give the largest contributions in relevant processes.
} 
A full list of the dimension-6 operators were initially presented by Buchmüller and Wyler [7], and also by Arzt et al. [8]. However, it was pointed out that some operators there are related with others through equations of motion, which means that they are not independent of each other [9]. Then, the whole related operators were rearranged to get rid of this redundancy in Refs. [10, 11].

Following the notation of Ref. [10], the relevant effective Lagrangian which describes non-standard interactions of the third generation for the parton-level process $q \bar{q} / g g \rightarrow t \bar{t} \rightarrow \ell^{+} X$ is given in [5] as

$$
\begin{aligned}
\mathcal{L}_{\mathrm{eff}}=\mathcal{L}_{t \bar{t} g, g g}+\mathcal{L}_{t b W}: & \\
\mathcal{L}_{t \bar{t} g, g g} & =-\frac{1}{2} g_{s} \sum_{a}\left[\bar{\psi}_{t}(x) \lambda^{a} \gamma^{\mu} \psi_{t}(x) G_{\mu}^{a}(x)\right. \\
& \left.-\bar{\psi}_{t}(x) \lambda^{a} \frac{\sigma^{\mu v}}{m_{t}}\left(d_{V}+i d_{A} \gamma_{5}\right) \psi_{t}(x) G_{\mu \nu}^{a}(x)\right] \\
\mathcal{L}_{t b W}= & -\frac{1}{\sqrt{2}} g\left[\bar{\psi}_{b}(x) \gamma^{\mu}\left(f_{1}^{L} P_{L}+f_{1}^{R} P_{R}\right) \psi_{t}(x) W_{\mu}^{-}(x)\right. \\
& \left.+\bar{\psi}_{b}(x) \frac{\sigma^{\mu v}}{M_{W}}\left(f_{2}^{L} P_{L}+f_{2}^{R} P_{R}\right) \psi_{t}(x) \partial_{\mu} W_{v}^{-}(x)\right]
\end{aligned}
$$

where $g_{s}$ and $g$ are the $S U(3)$ and $S U(2)$ coupling constants, $P_{L / R} \equiv\left(1 \mp \gamma_{5}\right) / 2, d_{V}, d_{A}$ and $f_{1,2}^{L, R}$ are form factors defined as

$$
\begin{array}{ll}
d_{V} \equiv \frac{\sqrt{2} v m_{t}}{g_{s} \Lambda^{2}} \operatorname{Re}\left(C_{u G \phi}^{33}\right), & d_{A} \equiv \frac{\sqrt{2} v m_{t}}{g_{s} \Lambda^{2}} \operatorname{Im}\left(C_{u G \phi}^{33}\right), \\
f_{1}^{L} \equiv V_{t b}+C_{\phi q}^{(3,33) *} \frac{v^{2}}{\Lambda^{2}}, & f_{1}^{R} \equiv C_{\phi \phi}^{33 *} \frac{v^{2}}{2 \Lambda^{2}}, \\
f_{2}^{L} \equiv-\sqrt{2} C_{d W}^{33 *} \frac{v^{2}}{\Lambda^{2}}, & f_{2}^{R} \equiv-\sqrt{2} C_{u W}^{33} \frac{v^{2}}{\Lambda^{2}},
\end{array}
$$

with $v$ being the Higgs vacuum expectation value and $V_{t b}$ being $(t b)$ element of Kobayashi-Maskawa matrix. In particular, $d_{V}$ and $d_{A}$ are the so-called chromomagnetic- and chromoelectric-dipole moments, respectively.

In the following calculations, we use the above effective Lagrangian for top-quark interactions and the usual standard-model Lagrangian for the other interactions which are not affected by top quarks hereafter.

\subsection{Optimal-observable analysis}

The optimal-observable analysis is a method that could systematically estimate the expected statistical uncertainties of measurable parameters [12-15]. Here we give a brief review of this procedure.

First, assume that we have a cross section

$$
\frac{d \sigma}{d \phi}(\equiv \Sigma(\phi))=\sum_{i} c_{i} f_{i}(\phi),
$$

where $f_{i}(\phi)$ are known functions of the final-state variables $\phi$ and $c_{i}$ 's are model-dependent coefficients. The goal is to determine the $c_{i}$ 's. This can be done by using appropriate weighting functions $w_{i}(\phi)$ such that $\int w_{i}(\phi) \Sigma(\phi) d \phi=c_{i}$. Then, we determine $w_{i}(\phi)$ as the next step. In general, different choices for $w_{i}(\phi)$ are possible, but there is a unique choice for which the resultant statistical error is minimized. Such functions are given by

$$
w_{i}(\phi)=\sum_{j} X_{i j} f_{j}(\phi) / \Sigma(\phi),
$$

where $X_{i j}$ is the inverse matrix of $\mathcal{M}_{i j}$ which is defined as

$$
\mathcal{M}_{i j} \equiv \int d \phi f_{i}(\phi) f_{j}(\phi) / \Sigma(\phi)
$$

Finally, using Eqs. (7) and (8), the statistical uncertainty of $c_{i}$ is obtained as

$$
\left|\delta c_{i}\right|=\sqrt{X_{i i} \sigma_{T} / N},
$$

where $\sigma_{T} \equiv \int(d \sigma / d \phi) d \phi$ and $N$ is the total number of events.

Let us apply this technique to the process $p p \rightarrow t \bar{t} X \rightarrow$ $\ell^{+} X^{\prime}$, expressing its differential cross section (the angular and energy distribution of the charged-lepton $\ell^{+}$) which we derived in [5] as follows:

$$
\begin{aligned}
\frac{d^{2} \sigma_{\ell}}{d E_{\ell} d \cos \theta_{\ell}}= & f_{\mathrm{SM}}\left(E_{\ell}, \cos \theta_{\ell}\right)+d_{V} f_{d_{V}}\left(E_{\ell}, \cos \theta_{\ell}\right) \\
& +d_{R} f_{d_{R}}\left(E_{\ell}, \cos \theta_{\ell}\right)+d_{V}^{2} f_{d_{V}^{2}}\left(E_{\ell}, \cos \theta_{\ell}\right) \\
& +d_{A}^{2} f_{d_{A}^{2}}\left(E_{\ell}, \cos \theta_{\ell}\right)+\cdots,
\end{aligned}
$$

where $f_{\mathrm{SM}}\left(E_{\ell}, \cos \theta_{\ell}\right)$ denotes the standard-model contribution, all the other $f_{I}\left(E_{\ell}, \cos \theta_{\ell}\right)$ describe the nonstandard model terms corresponding to their coefficients, and $d_{R}$ is defined as

$$
d_{R} \equiv \frac{M_{W}}{m_{t}} \operatorname{Re}\left(f_{2}^{R}\right)
$$

The explicit forms of $f_{I}\left(E_{\ell}, \cos \theta_{\ell}\right)$ at the parton level are easily found in the relevant formulas in [5].

Since the magnitude of $d_{V}$ and $d_{A}$ has been shown small $[5,6]$, we neglect any contribution from terms quadratic (or higher) in those non-standard model parameters hereafter. ${ }^{2}$ Note that all $d_{A}$ contributions disappear under this linear approximation, because there is no term proportional to $d_{A}$. Thus, the angular and energy distributions of a decay lepton are written as

$$
\begin{aligned}
\frac{d \sigma_{\ell}}{d \cos \theta_{\ell}} & =g_{1}\left(\cos \theta_{\ell}\right)+d_{V} g_{2}\left(\cos \theta_{\ell}\right), \\
\frac{d \sigma_{\ell}}{d E_{\ell}} & =h_{1}\left(E_{\ell}\right)+d_{V} h_{2}\left(E_{\ell}\right)+d_{R} h_{3}\left(E_{\ell}\right),
\end{aligned}
$$

where $g_{i}\left(\cos \theta_{\ell}\right)$ and $h_{i}\left(E_{\ell}\right)$ are given by

$$
\begin{aligned}
g_{i}\left(\cos \theta_{\ell}\right) & =\int d E_{\ell} f_{I}\left(E_{\ell}, \cos \theta_{\ell}\right), \\
h_{i}\left(E_{\ell}\right) & =\int d \cos \theta_{\ell} f_{I}\left(E_{\ell}, \cos \theta_{\ell}\right)
\end{aligned}
$$

with $i=1,2$ and 3 corresponding to $I=\mathrm{SM}, d_{V}$ and $d_{R}$, respectively. Here should be one comment about the

\footnotetext{
${ }^{2}$ The quadratic terms of $f_{1,2}^{L, R}$ have already been neglected in our previous papers. Indeed, studies at the Tevatron suggest that those contributions are tiny: See [16] for the latest data.
} 
angular distribution: We can thereby probe exclusively the $d_{V}$ term, since any contribution from $d_{R}$ disappears within our approximation as a result of the decoupling theorem found in [17-19].

We are now ready to calculate the following matrices:

$$
\begin{aligned}
M_{i j}^{c} & \equiv \int d \cos \theta_{\ell} \frac{g_{i}\left(\cos \theta_{\ell}\right) g_{j}\left(\cos \theta_{\ell}\right)}{g_{1}\left(\cos \theta_{\ell}\right)} \quad(i, j=1,2), \\
M_{i j}^{E} & \equiv \int d E_{\ell} \frac{h_{i}\left(E_{\ell}\right) h_{j}\left(E_{\ell}\right)}{h_{1}\left(E_{\ell}\right)} \quad(i, j=1,2,3)
\end{aligned}
$$

and their inverse matrices $X_{i j}^{c, E}$, all of which are apparently symmetric. Then the statistical uncertainties for the measurements of couplings $d_{V}$ and $d_{R}$ could be estimated by

$$
\left|\delta d_{V}\right|=\sqrt{X_{22}^{c} \sigma_{\ell} / N_{\ell}}=\sqrt{X_{22}^{c} / L}
$$

through the angular distribution, and

$$
\begin{aligned}
& \left|\delta d_{V}\right|=\sqrt{X_{22}^{E} \sigma_{\ell} / N_{\ell}}=\sqrt{X_{22}^{E} / L}, \\
& \left|\delta d_{R}\right|=\sqrt{X_{33}^{E} \sigma_{\ell} / N_{\ell}}=\sqrt{X_{33}^{E} / L}
\end{aligned}
$$

via the energy distribution, where $\sigma_{\ell}, N_{\ell}$ and $L$ denote the total cross section, the number of events and the integrated luminosity for the process, respectively.

\section{Numerical results and discussion}

Below we show the elements of $M^{c, E}$ computed for $\sqrt{s}=$ $7,8,10$, and $14 \mathrm{TeV}$, assuming $m_{t}=173 \mathrm{GeV}$ [20].

(1) The angular distribution

$$
\begin{aligned}
& (\mathbf{1 - 1}) \sqrt{s}=7 \mathrm{TeV} \\
& M_{11}^{c}=23.102, \quad M_{12}^{c}=-245.412, \quad M_{22}^{c}=2607.340 . \\
& (\mathbf{1 - 2}) \sqrt{s}=8 \mathrm{TeV} \\
& M_{11}^{c}=33.234, \quad M_{12}^{c}=-353.598, \quad M_{22}^{c}=3762.753 .
\end{aligned}
$$

(1-3) $\sqrt{s}=10 \mathrm{TeV}$

$$
M_{11}^{c}=59.333, \quad M_{12}^{c}=-632.179, \quad M_{22}^{c}=6736.735 .
$$

(1-4) $\sqrt{s}=14 \mathrm{TeV}$

$M_{11}^{c}=134.052, \quad M_{12}^{c}=-1428.300, \quad M_{22}^{c}=15220.286$.

(2) The energy distribution

$$
\begin{array}{lll}
\text { (2-1) } \sqrt{s}=7 \mathrm{TeV} & \\
M_{11}^{E}=23.102, & M_{12}^{E}=-245.412, & M_{13}^{E}=0.000 \\
M_{22}^{E}=2607.658, & M_{23}^{E}=-1.974, & M_{33}^{E}=15.323 .
\end{array}
$$

(2-2) $\sqrt{s}=8 \mathrm{TeV}$

$$
\begin{array}{lll}
M_{11}^{E}=33.234, & M_{12}^{E}=-353.598, & M_{13}^{E}=0.000, \\
M_{22}^{E}=3763.252, & M_{23}^{E}=-2.880, & M_{33}^{E}=21.124 .
\end{array}
$$

(2-3) $\sqrt{s}=10 \mathrm{TeV}$

$$
\begin{array}{lll}
M_{11}^{E}=59.333, & M_{12}^{E}=-632.179, & M_{13}^{E}=0.000, \\
M_{22}^{E}=6737.696, & M_{23}^{E}=-5.120, & M_{33}^{E}=35.233 .
\end{array}
$$

(2-4) $\sqrt{s}=14 \mathrm{TeV}$

$$
\begin{array}{lll}
M_{11}^{E}=134.052, & M_{12}^{E}=-1428.300, & M_{13}^{E}=0.000, \\
M_{22}^{E}=15222.443, & M_{23}^{E}=-10.953, & M_{33}^{E}=72.264 .
\end{array}
$$

Here, all $M_{13}^{E}$ became zero for the same reason as vanishing $d_{R}$ terms in the angular distribution, i.e., the decoupling theorem [17-19]. Using the inverse matrices calculated from the above elements and Eq.(16) - Eq.(18), we can estimate the statistical uncertainties of the relevant couplings.

Before giving the results, however, we should comment about instabilities of inverse-matrix computations: We noticed that the results fluctuate to a certain extent (beyond our expectation) depending on to which decimal places of $M^{c, E}$ we take into account as our input data. Therefore we calculate $X^{c, E}$ for the above $M^{c, E}$ and also for what are obtained by rounding those $M^{c, E}$ off to two decimal places. We then use their mean values to get $\delta d_{V}$ and $\delta d_{R}$ with "errors", which are the differences between the mean values and the maximum/minimum.

Let us explain these treatments more specifically by taking (1-1) as an example: For those different inputs, we get

$$
\begin{aligned}
\sqrt{X_{22}^{c}} & =1.73 \text { for } M_{11}^{c}=23.102, \cdots, \\
& =2.57 \text { for } M_{11}^{c}=23.10, \cdots,
\end{aligned}
$$

which lead to the mean value 2.15 , the maximum 2.57 , and the minimum 1.73 , and resultant errors \pm 0.42 , which are derived by $2.57-2.15$ and $1.73-2.15$.

We also have to explain how we can take into account QCD higher-order corrections: All the numerical computations of $M_{i j}^{c, E}$ in this section were done with the tree-level formulas. In order to include QCD corrections there, we multiply the tree cross sections by the $K$-factor. This factor disappears in the combination $X_{i i}^{c, E} \sigma_{\ell}$ and remains only in $N_{\ell}\left(=L \sigma_{\ell}\right)$ when we estimate $\delta d_{V, R}$. Therefore the luminosity $L$ discussed in the following should be understood as an effective one including $K$ (and also the lepton detection efficiency $\epsilon_{\ell}$ ).

We now show the whole results in Table 1 and Table 2 for the angular and energy distributions, respectively. The uncertainties we encountered here are hard to eliminate, but still the results will tell us the necessary luminosity for reaching the precision which we aim to realize. For example, we need at least $L \simeq 1500 \mathrm{pb}^{-1}\left(=1.5 \mathrm{fb}^{-1}\right)$ in order to achieve $\left|\delta d_{V}\right| \simeq O\left(10^{-2}\right)$ in case of measuring the angular distribution at the LHC whose colliding energy is $8 \mathrm{TeV}$. As mentioned, those $L$ should be divided by $K \simeq$ 1.5 and $\epsilon_{\ell}$. If we assume $\epsilon_{\ell}=0.5$, the resultant $L$ increases slightly, which however hardly affects our conclusion. 
Table 1. Estimated statistical uncertainties of $d_{V}$ from the angular distribution of a decay lepton.

\begin{tabular}{cc}
\hline$\sqrt{s}[\mathrm{TeV}]$ & $\left|\delta d_{V}\right| \times \sqrt{L}$ \\
\hline 7 & $2.15 \pm 0.42$ \\
8 & $2.25 \pm 0.96$ \\
10 & $1.12 \pm 0.12$ \\
14 & $0.73 \pm 0.02$ \\
\hline
\end{tabular}

Table 2. Estimated statistical uncertainties of $d_{V}$ and $d_{R}$ from the energy distribution of a decay lepton.

\begin{tabular}{cll}
\hline$\sqrt{s}[\mathrm{TeV}]$ & $\left|\delta d_{V}\right| \times \sqrt{L}$ & $\left|\delta d_{R}\right| \times \sqrt{L}$ \\
\hline 7 & $1.86 \pm 0.28$ & $0.35 \pm 0.02$ \\
8 & $1.70 \pm 0.51$ & $0.32 \pm 0.05$ \\
10 & $0.98 \pm 0.08$ & $0.22 \pm 0.01$ \\
14 & $0.65 \pm 0.02$ & 0.15 \\
\hline
\end{tabular}

\section{Summary}

Using a scenario of Buchmüller and Wyler [7] and the optimal-observable analysis, we studied the statistical significance of possible non-standard top-gluon couplings corresponding to $d_{V, A}$ and top- $W$ coupling corresponding to $d_{R}$ as model-independently as possible for the current and future LHC experiments.

Since it has been known that those non-standard couplings are not so large $[5,6],{ }^{3}$ we adopted a linear approximation for performing the optimal-observable analysis. Although the $d_{A}$ terms disappeared under this approximation, we got the following knowledge about the determination accuracy of $d_{V}$ and $d_{R}$ :

- Measuring the energy distribution could study both $d_{V}$ and $d_{R}$ at the same time with a higher precision than the case of the angular distribution.

- Since the angular distribution is affected by only $d_{V}$ contributions, this one is suitable for exploring the mechanism of top-pair productions exclusively though the precision of $d_{V}$ is slightly lower than that from the energy distribution.

Furthermore, it was pointed out that there were some ambiguities in inverse-matrix computations depending on where to round the input data (i.e., $M_{i j}^{c, E}$-elements) off, and consequently estimated statistical errors were affected thereby to a certain extent. However, our conclusion about the necessary luminosity and new-physics scale $\Lambda$ do not receive that serious influence, because those ambiguities do not change the order of the results.

Finally, if the LHC is going to be steadily upgraded and $L=500 \mathrm{fb}^{-1}$ is achieved at $\sqrt{s}=14 \mathrm{TeV}, d_{V}$ and $d_{R}$ could be determined with $\left|\delta d_{V}\right| \sim O\left(10^{-3}\right)$ and $\left|\delta d_{R}\right| \sim$ $O\left(10^{-4}\right)$ respectively from the energy distribution: Here, $\left|\delta d_{V}\right| \sim 0.001\left(\left|\delta d_{R}\right| \sim 0.0001\right)$ means that the contribution from effective operator $O_{u G \phi}^{33}\left(O_{u W}^{33}\right)$ is suppressed by $\Lambda \gtrsim$

\footnotetext{
${ }^{3}$ It is expected that results of LHC experiments with $\sqrt{s}=8 \mathrm{TeV}$ will give stronger constraints for $d_{V, A}[21]$.
}

7 (20) TeV if the center value of measured $d_{V}\left(d_{R}\right)$ is close to zero and $C_{u G \phi}^{33}\left(C_{u W}^{33}\right) \sim 1$, which we estimated from Eqs.(5) and (11).

On the other hand, concerning $d_{A}$ terms, which were neglected in this work, they induce CP-violating interactions, therefore those effects would be better off being probed via some kind of asymmetric observables [22].

\section{Acknowledgments}

This work was partly supported by the Grant-in-Aid for Scientific Research No. 22540284 from the Japan Society for the Promotion of Science. Part of the algebraic and numerical calculations were carried out on the computer system at Yukawa Institute for Theoretical Physics (YITP), Kyoto University.

\section{References}

[1] F. Abe et al. (CDF Collaboration), Phys. Rev. Lett. 74 (1995) 2626 (hep-ex/9503002).

[2] S. Abachi et al. (D0 Collaboration), Phys. Rev. Lett. 74 (1995) 2632 (hep-ex/9503003).

[3] For recent review J.F. Kamenik, J. Shu and J. Zupan, arXiv:1107.5257 [hep-ph].

[4] Z. Hioki and K. Ohkuma, Eur. Phys. J. C 65 (2010) 127 (arXiv:0910.3049 [hep-ph]); Eur. Phys. J. C 71 (2011) 1535 (arXiv:1011.2655 [hep-ph]).

[5] Z. Hioki and K. Ohkuma, Phys. Rev. D 83 (2011) 114045 (arXiv:1104.1221 [hep-ph]).

[6] Z. Hioki and K. Ohkuma, Phys. Lett. B 716 (2012) 310 (arXiv:1206.2413 [hep-ph]).

[7] W. Buchmuller and D. Wyler, Nucl. Phys. B 268 (1986) 621.

[8] C. Arzt, M.B. Einhorn and J. Wudka, Nucl. Phys. B 433 (1995) 41 (hep-ph/9405214).

[9] B. Grzadkowski, Z. Hioki, K. Ohkuma and J. Wudka, Nucl. Phys. B 689 (2004) 108 (hep-ph/0310159).

[10] J.A. Aguilar-Saavedra, Nucl. Phys. B 812 (2009) 181 (arXiv:0811.3842 [hep-ph]); Nucl. Phys. B 821 (2009) 215 (arXiv:0904.2387 [hep-ph]).

[11] B. Grzadkowski, M. Iskrzynski, M. Misiak and J. Rosiek, JHEP 1010 (2010) 085 (arXiv:1008.4884 [hep-ph]).

[12] D. Atwood and A. Soni, Phys. Rev. D 45 (1992) 2405.

[13] M. Davier, L. Duflot, F. Le Diberder and A. Rouge, Phys. Lett. B 306 (1993) 411.

[14] M. Diehl and O. Nachtmann, Z. Phys. C 62 (1994) 397.

[15] J.F. Gunion, B. Grzadkowski and X.-G. He, Phys. Rev. Lett. 77 (1996) 5172 (hep-ph/9605326).

[16] T. Aaltonen et al. [CDF and D0 Collaborations], Phys. Rev. D 85 (2012) 071106 (arXiv:1202.5272 [hepex]).

[17] B. Grzadkowski and Z. Hioki, Phys. Lett. B 476 (2000) 87 (hep-ph/9911505); Phys. Lett. B 529 (2002) 
82 (hep-ph/0112361); Phys. Lett. B 557 (2003) 55 (hep-ph/0208079).

[18] S.D. Rindani, Pramana 54 (2000) 791 (hepph/0002006).

[19] R.M. Godbole, S.D. Rindani and R.K. Singh, JHEP 0612 (2006) 021 (hep-ph/0605100).
[20] O. Brandt [CDF and D0 Collaborations], arXiv:1204.0919 [hep-ex].

[21] Z. Hioki and K. Ohkuma, in preparation.

[22] G. Valencia, PoS HQL 2012 (2012) 050 (arXiv:1301.0962 [hep-ph]). 